\title{
Optical trapping with a perfect vortex beam
}

\author{
Mingzhou Chen ${ }^{a}$, Michael Mazilu ${ }^{a}$, Yoshihiko Arita ${ }^{a}$, Ewan M. Wright ${ }^{b}$ and Kishan Dholakia $^{a}$ \\ ${ }^{a}$ SUPA, School of Physics \& Astronomy, University of St Andrews, North Haugh, St Andrews, \\ KY16 9SS, United Kingdom; \\ ${ }^{b}$ College of Optical Sciences, The University of Arizona, 1630 East University Boulevard, \\ Tuscon, Arizona 85721-0094, USA
}

\begin{abstract}
Vortex beams with different topological charge usually have different profiles and radii of peak intensity. This introduces a degree of complexity the fair study of the nature of optical OAM (orbital angular momentum). To avoid this, we introduced a new approach by creating a perfect vortex beam using an annular illuminating beam with a fixed intensity profile on an SLM that imposes a chosen topological charge. The radial intensity profile of such an experimentally created perfect vortex beam is independent to any given integer value of its topological charge. The well-defined OAM density in such a perfect vortex beam is probed by trapping microscope particles. The rotation rate of a trapped necklace of particles is measured for both integer and non-integer topological charge. Experimental results agree with the theoretical prediction. With the flexibility of our approach, local OAM density can be corrected in situ to overcome the problem of trapping the particle in the intensity hotspots. The correction of local OAM density in the perfect vortex beam therefore enables a single trapped particle to move along the vortex ring at a constant angular velocity that is independent of the azimuthal position. Due to its particular nature, the perfect vortex beam may be applied to other studies in optical trapping of particles, atoms or quantum gases.
\end{abstract}

Keywords: Optical Trapping, perfect vortex, Orbital Angular Momentum (OAM)

\section{INTRODUCTION}

In the optical domain, an optical vortex beam may possess orbital angular momentum (OAM) ${ }^{1}$ which is attributable to the helical wavefront of the beam. The number of interwinding helices (or the number of $2 \pi$ phase changes around the beam axis) is corresponding to the topological charge $\ell$. Such a vortex beam typically has a radial dependence upon the value of the topological charge. ${ }^{2-4}$ There have been a number of approaches to control the spatial property of a vortex beam using the Laguerre-Gaussian (LG) modes and high-order Bessel beams. In particular, Schmits and et. al. ${ }^{5}$ demonstrated the unchanged beam profile for different $\ell$ by interfering the two oppositely charged vortex beams. However, because of interference of the two beams, intensity modulation on the ring was unavoidable. Čižmár et. al. implemented an incoherent mode superposition to demonstrate smooth tuning of the OAM density ${ }^{6}$ with intensity variations though the use of an acousto-optic deflector may cause unwanted aberrations. In the context of all of these studies, a vortex beam with a fixed profile and continuous tuneable OAM density would be of significant interest.

The concept of a 'perfect optical vortex' was first introduced in Ref. ${ }^{7}$ denoting a vortex field whose intensity profile and radius would be independent of topological charge $\ell$. A particle trapped on such a beam would then rotate at a constant velocity set by the beam intensity, radius of the ring, and the topological charge. The dynamics of trapped particles in such a 'perfect' vortex beam was reported recently. ${ }^{8}$ Here, we will expound the details of optical trapping with such a 'perfect' vortex beam. In particular, we detail how we probe the local OAM density with a single trapped particle. In situ, we improve the beam quality in order to attain a uniform OAM density. 


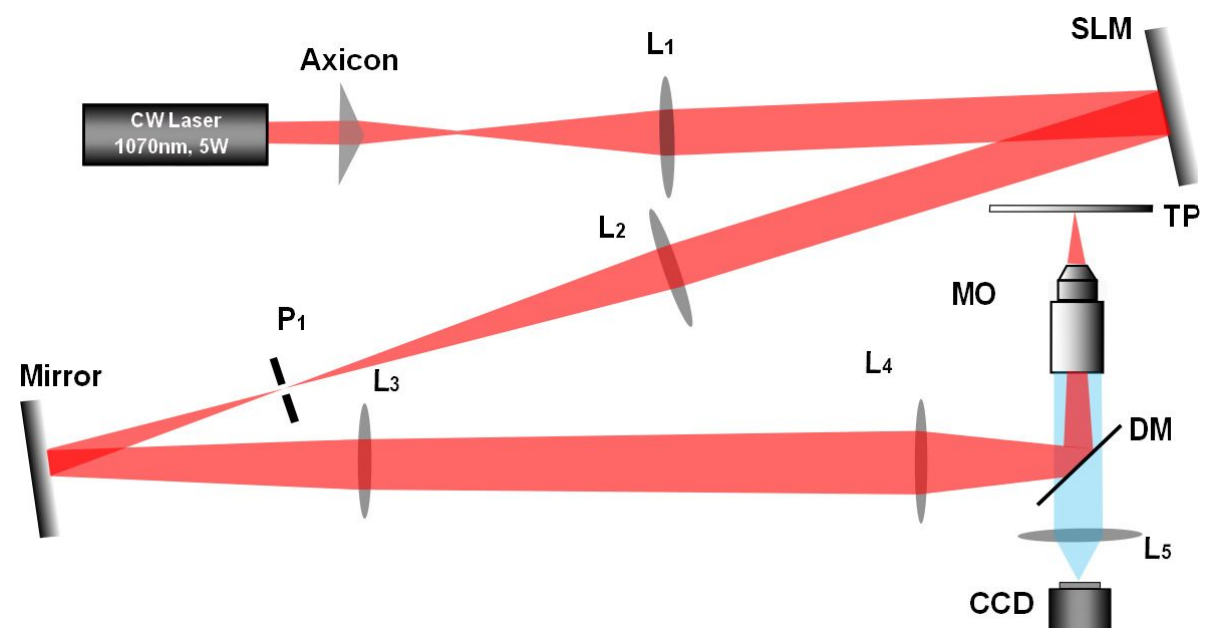

Figure 1. Optical setup used for trapping with a perfect vortex beam.

\section{EXPERIMENTAL SETUP AND BEAM CORRECTION}

In the experimental setup as shown in Fig. 1, an axicon (apex angle is $178^{\circ}$ ) together with a lens $\left(L_{1}\right)$ provide a ring illumination on the spatial light modulator (Hamamatsu LCOS-SLM X10468-03). In contrast to usually taking its Fourier transform, the SLM plane is directly imaged onto the trapping plane (TP) with lenses $L_{2} \sim$ $L_{4}$ and a microscope objective ( $M O$, Nikon, $40 \mathrm{X}, \mathrm{NA}=1.3$, oil). The modulated first diffraction order is selected by a pinhole $\left(P_{1}\right)$. A dichroic mirror $(D M)$ reflects the laser while transmitting the illumination light. The standard part of LED white light illumination used to illuminate the trapping plane is not shown in the Fig. 1 while the optical path is shown by the blue beam.

The topological charge of the beam is controlled by the vortex phase together with a grating phase applied on the SLM. The achievable topological charge is mainly constrained by the back aperture of $M O$ due to the ring illumination used. In our system, we can realise topological charges ranging from $\ell=-40$ to +40 . As most of optical aberrations in the system is attributable to the flatness of SLM surface, they can be corrected effectively by applying a well-known wavefront correction method. ${ }^{9}$ As examples shown by Fig.2 (a) and (b), the beam quality is improved after wavefront correction on SLM.

After wavefront correction, there are still some intensity hot spots in the beam profile (Fig. 2 (c)), which may cause the particles to be trapped in these local intensity maxima. Recalling the SLM is on the image plane of trapping plane and it is a phase-only one, we can remove these intensity hot spots locally by reducing the local efficiency of phase modulation at the locations of hot spots. After such an intensity profile correction, the intensity uniformity of the beam is significantly improved as can be seen in Fig. 2 (d).

\section{PARTICLES TRAPPING WITH A PERFECT VORTEX BEAM}

\subsection{Trapping a necklace of particles}

For the first studies, the created perfect vortex beam is filled with 26 polymer particles $(3 \mu m$ in diameter) to make a necklace as shown by Fig. 3 (a). This necklace of particles are rotating in two dimensions and pushed against the microscope coverslip surface of the water chamber. The roation of the particles can be dictated by the gradient and scattering forces resulting from the vortex beam.

To analyse the rotation rate of the particles, videos are recorded by a fast camera at a rate of $150 \mathrm{fps}$. We vary the topological charge $\ell$ from -35 to +35 in steps of 5 . From positions of one trapped particle in each video frame, the orbital rotation rate of the necklace can be evaluated. As shown by the fitted lines in Fig. 3

Further author information: (Send correspondence to Mingzhou Chen) Mingzhou Chen: mc225@st_andrews.ac.uk 


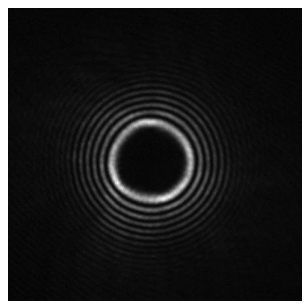

(a)

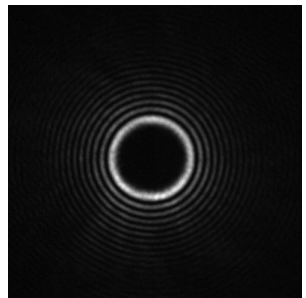

(b)

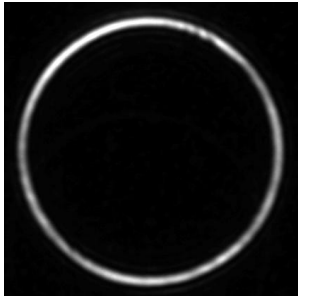

(c)

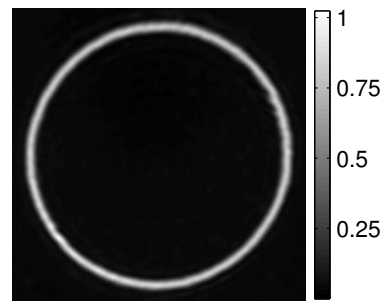

(d)

Figure 2. High order bessel beams $(\ell=20)$ on the focal plane of SLM before (a) and after $(b)$ wavefront correction.Vortex beams $(\ell=20)$ on the trapping plane before $(c)$ and after $(d)$ amplitude correction.
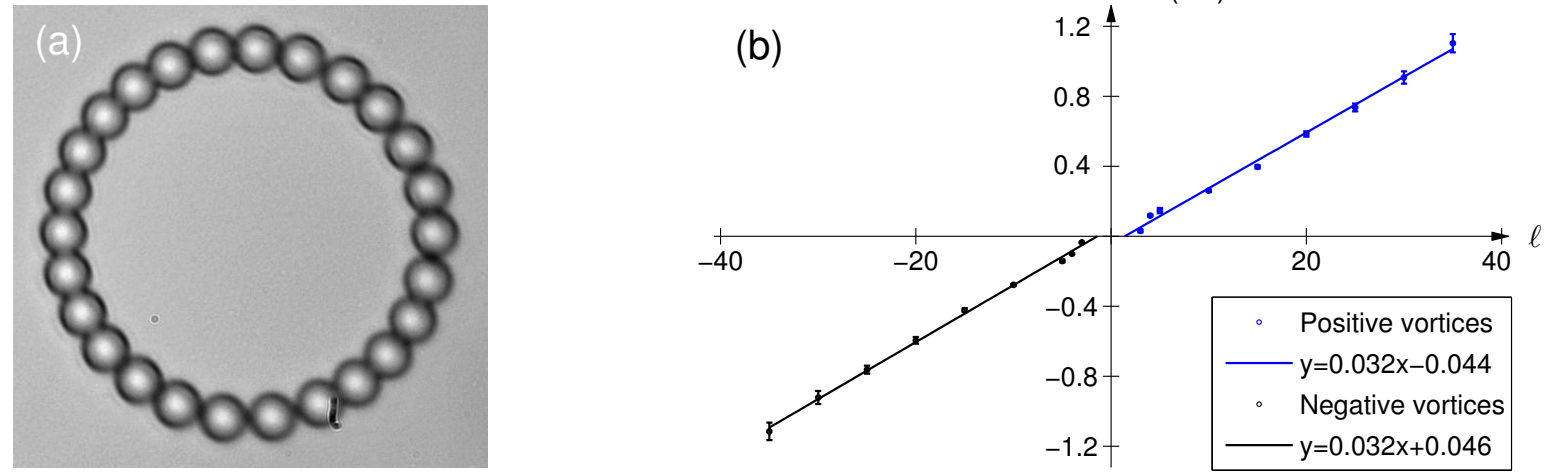

Figure 3. (a) A necklace of 26 polymer particles (3 $\mu \mathrm{m}$ in diameter) trapped by a he perfect vortex beam $\ell=20$. (b) The necklace rotation rate as a function of the integer topological charge $\ell$. Positive rotation rate is defined as anti-clockwise rotation. The solid lines represent a least-square linear regression of the original data.

(b), a linear relationship between the rotation rate and the topological charge of the perfect vortex beams can be clearly seen for both negative and positive topological charges due to the well-defined OAM density in each beam. The discontinuity near the origin (zero rotation rate) can be explained by the friction force between the particles and the glass coverslip.

Interestingly, the same approach is used to probe the OAM of vortex beams with a non-integer topological charge. With a necklace of 26 particles a collective 'averaged' rotation of the particle necklace can be observed. The rotation rate is measured when the necklace is rotating on a perfect vortex beam with the topological charge $\ell$ ranging from -12 to -9 and 9 to 12 in steps of $\Delta \ell=0.2$. The rotation rate does not vary linearly for fractional variations of the topological charge. However, it is in good agreement with the theoretical models ${ }^{10}$ as shown by the fitted soid lines in Fig. 4.

\subsection{Single particle trapping}

For the second studies, we load a single particle onto the vortex ring. A single trapped particle (a same $3 \mu \mathrm{m}$ polymer particle) can move along the vortex ring continuously. From its trajectory shown by the blue trace in Fig. 5 (a), a circle function can be fitted to estimate the rotation centre which is denoted by the blue circle in Fig. 5 (a). The distribution of particle positions on the ring is also shown in Fig. 5 (b). These two Gaussian peaks indicate that our vortex ring is not a perfect circle but with an ellipsity of 2 pixels. Similarly from positions of this single trapped particle in each video frame, the angular speed of this particle can be estimated as shown in Fig. 5 (c). Obviously, the angular velocity of such a trapped particle is not very uniform on the ring due to the local variations of OAM density. At some locations as pointed by the black arrows in Fig. 5 (c), the angular velocity even drops down to a negative value when the particle motion is mainly dominated by the Brownian motion. 

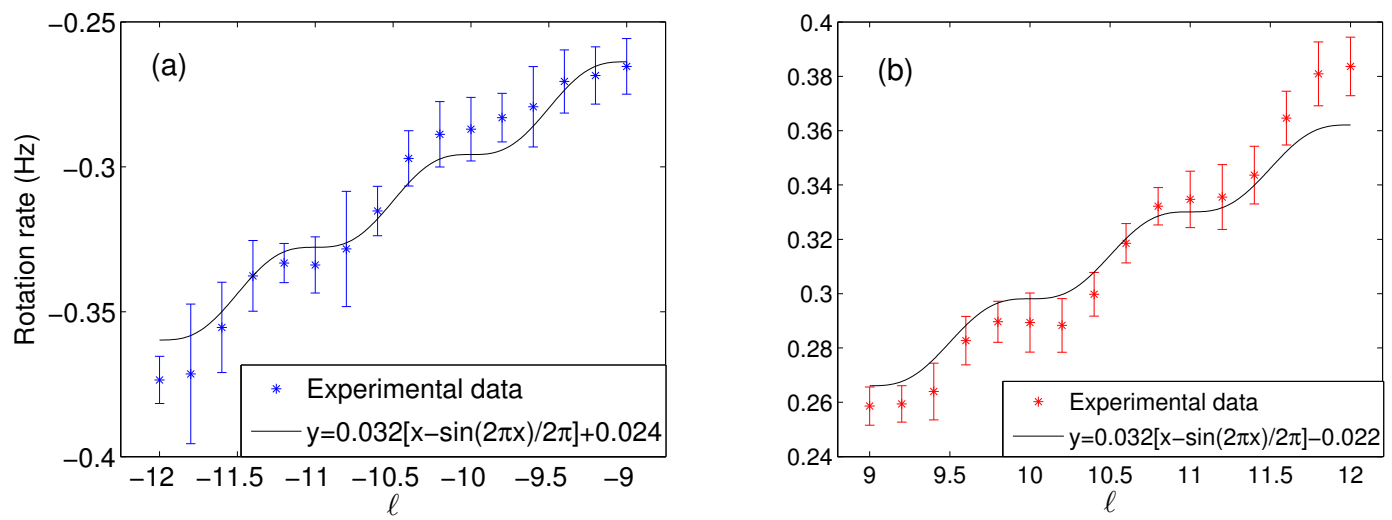

Figure 4. The particle rotation rate as a function of both positive (a) andthe necklace negative (b) fractional topological charge $\ell$. The solid lines with a specific prefactor and offset shows a least-square regression of the experimental data to the theoretical prediction of OAM per photon of a fractional vortex as $\ell-\sin (2 \pi \ell) /(2 \pi) .{ }^{10}$
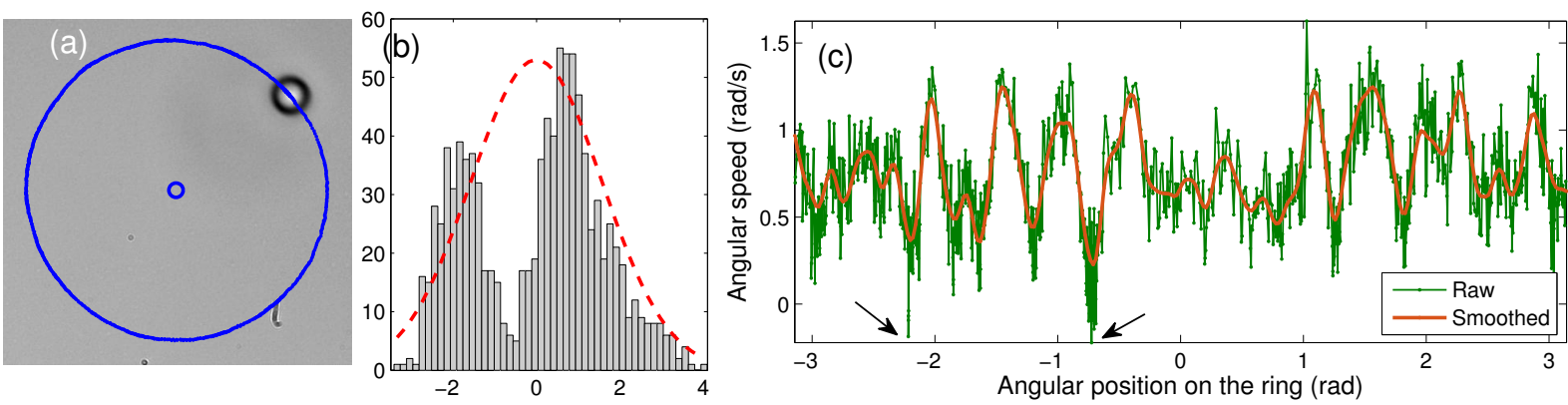

Figure 5. (a) a video frame of a single trapped particle. (b) Histogram of particle distribution along the radial direction on the vortex ring. (c) Angular speeed of the trapped partile along the vortex ring.

The variations in the angular speed of a single trapped particle in the perfect vortex beam can only be explained by the inpure orbital angular momentum state. This statement can be verified simply by a numerical simulation of optical force on a vortex ring as shown in Fig.6. A uniform optical force along the perfect vortex ring can only be achieved when the beam is in a pure orbital angular momentum state which in the perfect case corresponds to integer topological charge.

Based on the knowledge that the particle rotation velocity is linearly dependent upon the OAM density, the angular velocity can be adjusted locally by increasing or decreasing the local phase gradient. This adjustment can be realised by the SLM since it directly controls the phase of the vortex beam. Where the angular velocity

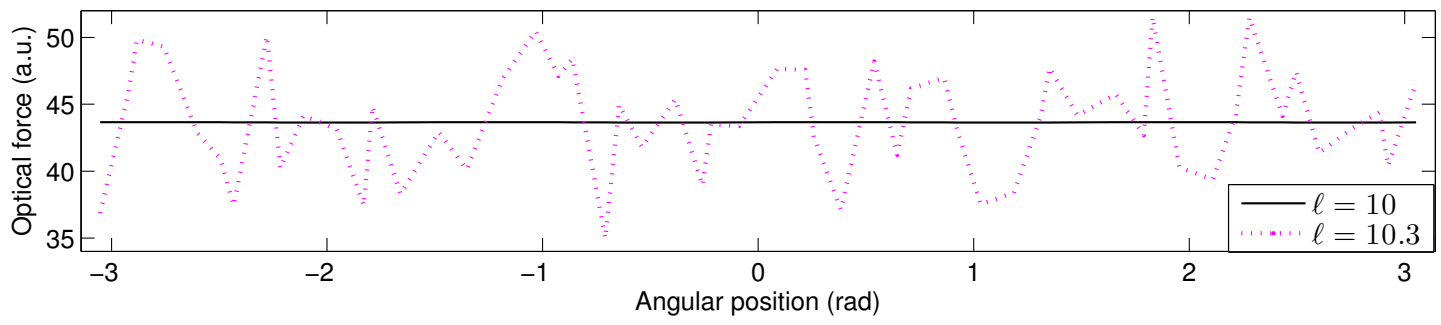

Figure 6. Numerical simulation of optical force on a vortex ring. 


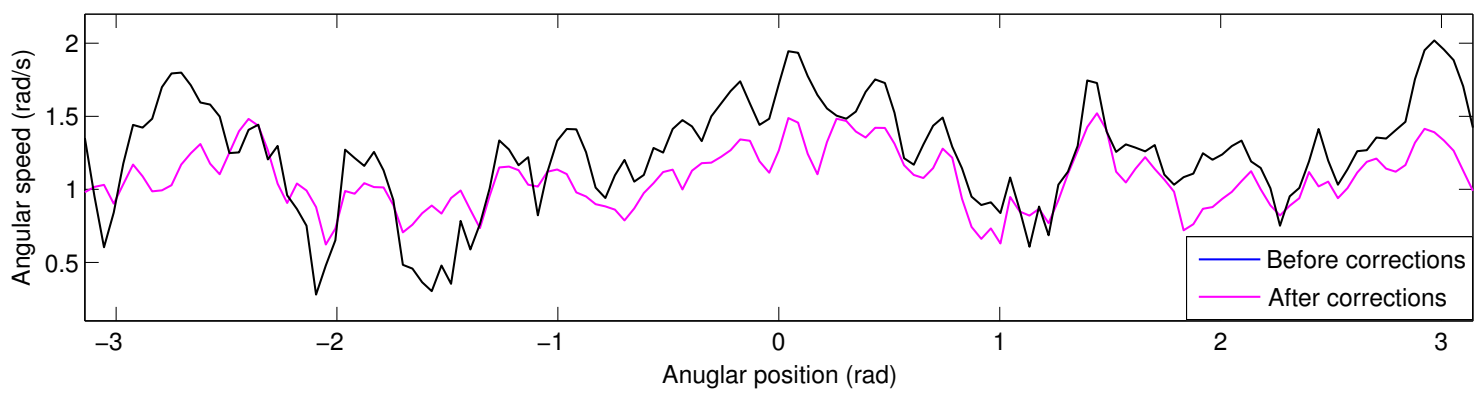

Figure 7. Angular speed of a single trapped particle on a perfect vortex beam.

is high, we can reduce the phase gradient and vice versa.

With the application of beam corrections discussed above, we can achieve smooth rotation speed for a single trapped particle on a perfect vortex beam, as shown in Fig. 7 .

\section{CONCLUSION}

In conclusion, we introduce an approach for generating perfect vortex beams with a uniform intensity profile regardless of its topological charge. Phase, amplitude and local OAM density correction are applied in situ to achieve a smooth particle motion. Such a perfect vortex beam may be used for other applications including micromixing and moving microparticles on complex trajectories.

\section{ACKNOWLEDGMENTS}

The authors would like to thank the UK Engineering and Physical Sciences Research Council (EPSRC) for funding.

\section{REFERENCES}

[1] Allen, L., Beijersbergen, M. W., Spreeuw, R. C., and Woerdman, J. P., "Orbital angular momentum of light and the transformation of Laguerre-Gaussian laser modes," Phys. Rev. A 45, 8185-8189 (June 1992).

[2] Simpson, N. B., Allen, L., and Padgett, M. J., "Optical tweezers and optical spanners with LaguerreGaussian modes," J. Mod. Opt. 43(12), 2485-2492 (1996).

[3] Garcés-Chávez, V., McGloin, D., Padgett, M. J., Dultz, W., Schmitzer, H., and Dholakia, K., "Observation of the transfer of the local angular momentum density of a multiringed light beam to an optically trapped particle," Phys. Rev. Lett. 91, 093602 (Aug 2003).

[4] Curtis, J. E. and Grier, D. G., "Structure of optical vortices," Phys. Rev. Lett. 90, 133901 (apr 2003).

[5] Schmitz, C. H. J., Uhrig, K., Spatz, J. P., and Curtis, J. E., "Tuning the orbital angular momentum in optical vortex beams," Opt. Express 14, 6604-6612 (jul 2006).

[6] Čižmár, T., Dalgarno, H. I. C., Ashok, P. C., Gunn-Moore, F. J., and Dholakia, K., "Interference-free superposition of non-zero order light modes: functionalized optical landscapes," Appl. Phys. Lett. 98, 081114 (2011).

[7] Ostrovsky, A. S., Rickenstorff-Parrao, C., and Arrizón, V., "Generation of the "perfect" optical vortex using a liquid-crystal spatial light modulator," Opt. Lett. 38, 534-536 (feb 2013).

[8] Chen, M., Mazilu, M., Arita, Y., Wright, E. M., and Dholakia, K., "Dynamics of microparticles trapped in a perfect vortex beam," Opt. Lett. 38(22), 4919-4922 (2013).

[9] Čižmár, T., Mazilu, M., and Dholakia, K., "In situ wavefront correction and its application to micromanipulation," Nat. Photonics 4, 388-394 (June 2010).

[10] Leach, J., Yao, E., and Padgett, M. J., "Observation of the vortex structure of a non-integer vortex beam," New J. Phys. 6, 71 (2004). 